\title{
Some Information on Quaternions Useful in Texture Calculations
}

\author{
A. MORAWIEC and J. POSPIECH \\ Institute for Metal Research, Polish Academy of Science, Cracow
}

(Received 19 July, 1988; in final form 6 October, 1988)

Crystal orientation is described by a rotation which can be expressed by a great number of different sets of parameters. Among these, the quaternion representation is the most economic one. The basic properties of quaternions are briefly summarized and their interrelationships with other orientation coordinates such as rotation axis and angle, the orientation matrix, and the Euler angles are given.

KEY WORDS: Quaternions, Representation of Orientations, Euler angles.

\section{INTRODUCTION}

In texture analysis the orientation is usually described by Euler angles, sometimes by rotation axis and rotation angle or other rotation parameters.

From among the possible orientation descriptions (see e.g. Goldstein (1953) or Korn and Korn, (1961)) the most economic one from the viewpoint of calculations seems to be the description by means of quaternions. The multiplication of four-element quaternions corresponding to the composition of the rotations requires considerably smaller number of operations and the relations of quaternion elements with the rotation parameters are in general simpler than they are in the case of elements of an orthogonal matrix. 
We shall recall the basic relations referring to the algebra of the quaternions and write down the relations which can be applied in certain calculation operations of texture analysis.

\section{QUATERNIONS}

The quaternion is a vector of a four-dimensional vector space $\mathbb{Q}$ with its basis $\left\{e_{\mu}: \mu=0,1,2,3\right\}$ over the real field in which the multiplication is defined by the following relations:

$$
\begin{gathered}
e_{0} e_{0}=e_{0}, \quad e_{0} e_{1}=e_{1} e_{0}=e_{1}, \quad e_{0} e_{2}=e_{2} e_{0}=e_{2}, \\
e_{0} e_{3}=e_{3} e_{0}=e_{3}, \quad e_{1} e_{2}=-e_{2} e_{1}=e_{3}, \quad e_{2} e_{3}=-e_{3} e_{2}=e_{1}, \\
e_{3} e_{1}=-e_{1} e_{3}=e_{2}, \quad e_{1} e_{1}=e_{2} e_{2}=e_{3} e_{3}=-e_{0} .
\end{gathered}
$$

The quaternion $x=x^{0} e_{0}+x^{1} e_{1}+x^{2} e_{2}+x^{3} e_{3}$ will be written briefly in the form $x=x^{\mu} e_{\mu}=x^{0} e_{0}+x^{i} e_{i}$, in which the summation symbol is omitted, the Greek indices run over the set $\{0,1,2,3\}$, while the Latin indices run over the set $\{1,2,3\}$. Formulae (1) may be written in a compact form as (Cornwell (1984))

$$
\begin{gathered}
e_{\mu} e_{0}=e_{0} e_{\mu}=e_{\mu}, \quad e_{i} e_{j}=\varepsilon_{i j k} e_{k}-\delta_{i j} e_{0} . \\
\delta_{i j}= \begin{cases}1, & \text { when } i=j \\
0, & \text { when } i \neq j\end{cases} \\
\varepsilon_{i j k}=\left\{\begin{aligned}
1, & \text { when }(i j k) \text { is even permutation of (123) } \\
-1, & \text { when }(i j k) \text { is odd permutation of (123) } \\
0, & \text { in the other cases }
\end{aligned}\right.
\end{gathered}
$$

Hence when $x=x^{\mu} e_{\mu}$ and $y=y^{\mu} e_{\mu}$ are two arbitrary quaternions their product is

$$
x y=\left(x^{0} y^{0}-x^{i} y^{i}\right) e_{0}+\left(x^{0} y^{k}+y^{0} x^{k}+\varepsilon_{i j k} x^{i} y^{j}\right) e_{k} .
$$

The quaternion $x^{*}=x^{0} e_{0}-x^{i} e_{i}$ is called a quaternion conjugate to $x=x^{\mu} e_{\mu}=x^{0} e_{0}+x^{i} e_{i}$. Furthermore we shall be interested in quaternions satisfying the condition $x x^{*}=e_{0}$. Their set will be denoted by $Q ; Q=\left\{x \in \mathbb{Q}: x x^{*}=e_{0}\right)$. It is easy to check that if $x, y \in Q$ then $x y \in Q$, and the condition $x x^{*}=e_{0}$ expressed by means of coordinates has the form $x^{\mu} x^{\mu}=1$. 
Significant is the fact of the existance of a surjective mapping $h: Q \rightarrow S O(3)$ such that $h(x y)=h(y) h(x)$ (see Altmann (1986)). There exists also a mapping $h^{\prime}: Q \rightarrow S O(3)$ defined as $h^{\prime}(x)=h\left(x^{*}\right)$ and satisfying the condition $h^{\prime}(x y)=h^{\prime}(x) h^{\prime}(y)$. For further consideration the first mapping, i.e. $h$ will be selected.

\section{THE RELATION OF THE QUATERNION WITH THE ORTHOGONAL MATRIX}

The mapping $h: Q \rightarrow S O(3)$ has the form

$$
g_{i j}=(h(x))_{i j}=\left(\left(x^{0}\right)^{2}-x^{k} x^{k}\right) \delta_{i j}+2 x^{i} x^{j}+2 \varepsilon_{i j k} x^{0} x^{k}
$$

where $g_{i j}$ are elements of the rotation matrix. Since $h(x)=h(-x)$, the quaternions $x$ and $-x$ represent the same rotation. Hence the coordinates will be selected in such a way that $x^{0}>0$ or when $x^{0}=0$ that $x^{1}>0$ etc.

Let us express the coordinates $x^{\mu}$ of the quaternion $x$ through the elements of the matrix $g$. Accepting $j=i$ in formula (4) summing over $i$ and utilizing $x^{\mu} x^{\mu}=1$ we shall find $g_{i i}=4\left(x^{0}\right)^{2}-1$, and hence

$$
x^{0}=\frac{\left(g_{i i}+1\right)^{1 / 2}}{2} .
$$

From the relation (4) there follows also $\varepsilon_{i j k} g_{j k}=4 x^{0} x^{i}$ and assuming that $x^{0} \neq 0$ we get

$$
x^{i}=\frac{\varepsilon_{i j k} g_{j k}}{2\left(g_{l l}+1\right)^{1 / 2}} \text {. }
$$

When $x^{0}=0$, then expression (4) takes the form of a system of equations $g_{i j}=2 x^{i} x^{j}-\delta_{i j}$ from which it is easy to find $x^{i}$.

\section{RELATION OF THE QUATERNION COORDINATES WITH THE ROTATION AXIS AND THE ROTATION ANGLE}

Since the rotation angle $\omega$ is connected with the trace of the rotation matrix by the relation $g_{i i}=2 \cos \omega+1$, we obtain from expression (5)

$$
x^{0}=\cos \frac{\omega}{2} \text {. }
$$


On the basis of expression (4) there is also $g_{i j} x^{j}=x^{i}$ and only when the coordinates $x^{i}$ are not simultaneously equal to zero, i.e. when $x^{i} x^{i} \neq 0$, then $x^{i}=\alpha n^{i}$, where $n^{i}$-coordinate of the rotation axis. However, the following relation holds: $1=x^{\mu} x^{\mu}=\left(x^{0}\right)^{2}+\alpha^{2} n^{i} n^{i}$. Since $n^{i} n^{i}=1$ on the basis of (7) we have: $\alpha= \pm \sin (\omega / 2)$. Calculating $x^{3}$ from (4) in the case when $x^{1}=x^{2}=n^{1}=n^{2}=0$ we find that the proper sign is + (the sign is opposite when accepting the mapping $h^{\prime}$ ). Hence

$$
x^{i}=n^{i} \sin \frac{\omega}{2} .
$$

Conversely, on the basis of (7) and (8) we get

$$
\begin{aligned}
& \cos \omega=\left(x^{0}\right)^{2}-x^{i} x^{i}=2\left(x^{0}\right)^{2}-1 \\
& \sin \omega=2 x^{0}\left(x^{i} x^{i}\right)^{1 / 2}=2 x^{0}\left(1-\left(x^{0}\right)^{2}\right)^{1 / 2},
\end{aligned}
$$

and, if $\omega \neq 0$

$$
n^{i}=x^{i}\left(1-\left(x^{0}\right)^{2}\right)^{-1 / 2}=x^{i}\left(x^{k} x^{k}\right)^{-1 / 2} .
$$

From formulae (7) and (8) one obtains directly the quaternion forms of the rational symmetry elements, e.g. for the four-fold axis $L_{[001]}^{4}$ we have $\frac{\sqrt{2}}{2}\left(e_{0}+e_{3}\right)$ or for the three-fold axis $L_{[111]}^{3}$ we have $\frac{1}{2}\left(e_{0}+e_{1}+e_{2}+e_{3}\right)$.

\section{RELATIONS OF THE QUATERNION COORDINATES WITH EULER ANGLES}

A rotation described by Euler angles $\varphi_{1}, \phi, \varphi_{2}$ is composed of three successive rotations of the coordinate system about the axis $z$ by an angle $\varphi_{1}$, about the axis $x$ by an angle $\phi$ and about the axis $z$ by an angle $\varphi_{2}$. To these rotations, on the basis of (7) and (8) correspond the following quaternions

$$
\cos \frac{\varphi_{1}}{2} e_{0}+\sin \frac{\varphi_{1}}{2} e_{3}, \quad \cos \frac{\phi}{2} e_{0}+\sin \frac{\phi}{2} e_{1}, \quad \cos \frac{\varphi_{2}}{2} e_{0}+\sin \frac{\varphi_{2}}{2} e_{3}
$$

Their product represents thus the rotation by Euler angles $\varphi_{1}, \phi$, 
$\varphi_{2}$. Hence we get

$$
\begin{aligned}
x^{0}=\cos \frac{\phi}{2} \cos \frac{\varphi_{1}+\varphi_{2}}{2}, & x^{1}=\sin \frac{\phi}{2} \cos \frac{\varphi_{1}-\varphi_{2}}{2}, \\
x^{2}=\sin \frac{\phi}{2} \sin \frac{\varphi_{1}-\varphi_{2}}{2}, & x^{3}=\cos \frac{\phi}{2} \sin \frac{\varphi_{1}+\varphi_{2}}{2} .
\end{aligned}
$$

Simple transformations of the above formulae allow to obtain the reverse relations

$$
\begin{aligned}
& \cos \phi=\left(\left(x^{0}\right)^{2}+\left(x^{3}\right)^{2}\right)-\left(\left(x^{1}\right)^{2}+\left(x^{2}\right)^{2}\right), \quad \sin \phi=2 \chi \\
& \cos \varphi_{1}=\left(x^{0} x^{1}-x^{2} x^{3}\right) / \chi, \quad \sin \varphi_{1}=\left(x^{0} x^{2}+x^{1} x^{3}\right) / \chi \\
& \cos \varphi_{2}=\left(x^{0} x^{1}+x^{2} x^{3}\right) / \chi, \quad \sin \varphi_{2}=\left(x^{1} x^{3}-x^{0} x^{2}\right) / \chi
\end{aligned}
$$

where

$$
\chi:=\left[\left(\left(x^{0}\right)^{2}+\left(x^{3}\right)^{2}\right)\left(\left(x^{1}\right)^{2}+\left(x^{2}\right)^{2}\right)\right]^{1 / 2} \neq 0 .
$$

When $\chi=0$ there is $x^{1}=x^{2}=0$ or $x^{0}=x^{3}=0$. In the first case we have

$$
\left.\phi=0, \quad \cos \left(\varphi_{1}+\varphi_{2}\right)=\left(x^{0}\right)^{2}-\left(x^{3}\right)^{2}, \quad \sin \varphi_{1}+\varphi_{2}\right)=2 x^{0} x^{3},
$$

in the other case

$$
\phi=\pi, \quad \cos \left(\varphi_{1}-\varphi_{2}\right)=\left(x^{1}\right)^{2}-\left(x^{2}\right)^{2}, \quad \sin \left(\varphi_{1}-\varphi_{2}\right)=2 x^{1} x^{2}
$$

\section{FINAL REMARKS}

The above considerations show that the quaternions may represent a convenient tool in computation operations of texture analysis. Use is made here of the fact that the operations which are performed on nine-element orthogonal matrices can be replaced in an equivalent manner by operations by four-element quaternions. The usage of quaternions simplifies also transformations of the rotation parameters of one kind to rotation parameters of another kind.

It should also be mentioned that the components $x^{1}, x^{2}, x^{3}$ of the quaternion may be used as rotation parameters. In the cartesian 
system the rotation is represented by a point of the sphere $\left(x^{1}\right)^{2}+\left(x^{2}\right)^{2}+\left(x^{3}\right)^{2} \leq 1$. (Antipodal points of the surface represent the same rotation.)

The formula for the volume element in the coordinates $x^{1}, x^{2}, x^{3}$ has the form $d g=\frac{1}{\pi^{2}} \frac{1}{x^{0}} d x^{1} d x^{2} d x^{3}$ where $x^{0}=\left(1-x^{k} x^{k}\right)^{1 / 2}$.

\section{References}

Altmann, S. L., Rotations, Quaternions and Double Groups, Calderon Press, Oxford, 1988.

Cornwell, J. F., Group Theory in Physics, Academic Press, London, 1984.

Goldstein, H., Classical Mechanics, Addison-Wesley, Reading, Mass., 1953.

Korn, G. A., Korn, T. M., Mathematical Handbook for Scientists and Engineers, McGraw-Hill, N.Y., 1961. 